\title{
Phase transitions related to the pigeonhole principle
}

\author{
Michiel De Smet* and Andreas Weiermann ${ }^{\star \star}$ \\ 1 Smidsestraat 192 \\ B 9000 Ghent \\ Belgium \\ mds@michieldesmet .eu \\ 2 Department of Mathematics \\ Ghent University \\ Building S22 \\ Krijgslaan 281 \\ B 9000 Ghent \\ Belgium \\ Andreas . Weiermann@UGent . be
}

\begin{abstract}
Since Jeff Paris introduced them in the late seventies [Par78], densities turned out to be useful for studying independence results. Motivated by their simplicity and surprising strength we investigate the combinatorial complexity of two such densities which are strongly related to the pigeonhole principle. The aim is to miniaturise Ramsey's Theorem for 1-tuples. The first principle uses an unlimited amount of colours, whereas the second has a fixed number of two colours. We show that these principles give rise to Ackermannian growth. After parameterising these statements with respect to a function $f: \mathbb{N} \rightarrow \mathbb{N}$, we investigate for which functions $f$ Ackermannian growth is still preserved.
\end{abstract}

Keywords Ackermann function, pigeonhole principle, Ramsey theory, phase transitions.

\section{Introduction}

The pigeonhole principle is one of the most well-know combinatorial principles, due to both its simplicity and usefulness. The principle is also known as the chest-of-drawers principle or Schubfachprinzip and is attributed to Dirichlet in 1834. The pigeonhole principle can also be considered as a finite instance of Ramsey's theorem for 1-tuples. So, if $\mathrm{RT}_{k}^{n}$ stands for Ramsey's Theorem for $n$ dimensions and $k$ colours, i.e.

$$
\begin{aligned}
\mathrm{RT}_{k}^{n} \leftrightarrow & \text { For every } G:[\mathbb{N}]^{n} \rightarrow k \text { there exists an infinite set } H \\
& \text { such that } G\left\lceil[H]^{n}\right. \text { is constant, }
\end{aligned}
$$

\footnotetext{
* Research supported in part by Fonds Wetenschappelijk Onderzoek (FWO) - Flanders

** Research supported in part by(FWO) and the John Templeton Foundation
} 
then the pigeonhole principle is a finite instance of $\mathrm{RT}_{<\infty}^{1}=\forall k \mathrm{RT}_{k}^{1}$. In this paper we will investigate miniaturisations of the statements $\mathrm{RT}_{<\infty}^{1}$ and $\mathrm{RT}_{2}^{1}$. Let us recall some results from reverse mathematics: for any fixed natural number $k, \mathrm{RCA}_{0} \vdash \mathrm{RT}_{k}^{1}$, whereas $\mathrm{WKL}_{0} \nvdash \mathrm{RT}_{<\infty}^{1}$. Both results are due to Hirst (see [Hir87], Theorem 6.3 and Theorem 6.5). In addition, there it is also proved that $\mathrm{RT}_{<\infty}^{1}$ does not imply $\mathrm{ACA}_{0}$ over $\mathrm{RCA}_{0}$. As it does not fit nicely into the programme of reverse mathematics, one might be tempted to think that $\mathrm{RT}_{<\infty}^{1}$ is of little importance. However, it pops up every now and then in the literature. It is, for instance, equivalent to Rado's Lemma over $\mathrm{RCA}_{0}$ (see [Hir87], Theorem $6.6)$.

For miniaturising $\mathrm{RT}_{<\infty}^{1}$ and $\mathrm{RT}_{2}^{1}$ we define two notions of density, $n$-density and $(\alpha, 2)$-density, which are parametrised by a function $f: \mathbb{N} \rightarrow \mathbb{N}$. Using these notions we define two first order assertions and study their provability with respect to $\mathrm{I} \Sigma_{1}$, the first-order part of $\mathrm{RCA}_{0}$.

We show which $f$ give rise to Ackermannian growth and determine the exact phase transition. In case of $n$-density Ackermannian growth is obtained for $f(i)=$

$i^{\frac{1}{A_{\omega}^{-1}(i)}}$, whereas for $f(i)=i^{\frac{1}{A_{d}^{-1}(i)}}$ it is not. Here $A_{d}$ denotes the $d$-th branch of the Ackermann function $A_{\omega}$. Our proof will show that in these results $A_{\omega}\left(A_{d}\right)$ could be replaced by any non decreasing unbounded non primitive recursive function (resp. by any non decreasing unbounded primitive recursive function). In the case of $(\alpha, 2)$-density we restrict ourselves to only two colours and strength disappears, as expected. Surprisingly, iterating up to $\omega^{2}$ suffices to gain proof theoretic strength again. It turns out that $f(i)=\frac{1}{A_{d}^{-1}(i)} \log (i)$ gives rise to no more than primitive recursive growth, but $f(i)=\frac{1}{A_{\omega}^{-1}(i)} \log (i)$ does. Our proof will show that also in these results $A_{\omega}\left(A_{d}\right)$ could be replaced by any non decreasing unbounded non primitive recursive function (resp. by any non decreasing unbounded primitive recursive function).

We would like to mention that the $n$-density threshold functions are exactly the same as those for the parameterised Kanamori-McAloon principle, whereas the $\left(\omega^{2}, 2\right)$-density functions equal those for the parameterised Paris-Harrington principle [KLOW08,WVH2012]. It is our hope that by investigating miniaturisations of $\mathrm{RT}_{<\infty}^{1}$ and $\mathrm{RT}_{2}^{2}$ one could obtain insights into the seemingly difficult question whether $\mathrm{RT}_{2}^{2}$ does or does not prove the totality of the Ackermann function (the so called Ramsey for pairs problem).

For related work we also we also refer to [DSW08] and the unpublished $\mathrm{PhD}$ thesis of the first author [DS11].

\section{$2 \quad n$-Density}

Henceforth, let $f: \mathbb{N} \rightarrow \mathbb{N}$ be any (elementary recursive function), such that $1 \leq f(x) \leq x$, for $x$ large enough. We define the functions $F_{f, k}$ and $F_{f}$, depending 
on $f$, by

$$
\begin{aligned}
F_{f, 0}(n) & :=n+1 \\
F_{f, k+1}(n) & :=\underbrace{F_{f, k}\left(\ldots \left(F_{f, k}\right.\right.}_{f(n) \text { times }}(n)) \ldots):=F_{f, k}^{f(n)}(n) \\
F_{f}(n) & :=F_{f, n}(n),
\end{aligned}
$$

for every $k, n \in \mathbb{N}$.

If it is clear which $f$ we are working with, we leave out the subscript $f$ and simply write $F_{k}$ and $F$, instead of $F_{f, k}$ and $F_{f}$, respectively. We also consider functions $f$ with non integer values. It is then understood that we round a value $f(i)$ down to $\lfloor f(i)\rfloor$, the biggest natural number below $f(i)$. Moreover we assume that $f$ has always values at least as big as 1 . It is easy to verify that the functions $F_{f, k}$ and $F_{f}$ are strictly monotonic increasing if the parameter function $f$ is non decreasing.

In case of $f(i)=i$ we write $A_{\omega}$ for $F_{f}$ and $A_{d}$ for $F_{f, d} . A_{\omega}$ is a standard choice for the well known Ackermann function, which is a recursive but not a primitive recursive function. The function $A_{d}$ is called the $d$-th branch of the Ackermann function. Every function $A_{d}$ is primitive recursive. In [OW09] a classification is given of those functions $f$ for which $F_{f}$ is primitive or non primitive recursive.

Let us define $n$-density, the first density notion related to the pigeonhole principle. In this case the number of colours depends on the minimum of $X$ and the function $f$.

Definition $1 X$ is called 0 -dense $(f)$ if $|X| \geq \max \{f(\min X), 3\} . X$ is called $(n+1)$-dense $(f)$ if for all $G: X \rightarrow f(\min X)$, there exists $Y \subseteq X$, such that $Y$ is homogeneous for $G$ and $Y$ is n-dense $(f)$.

Lemma 1 Assume that $k \leq l$ and that $X \subseteq[k, l]$ and that $f, g:[k, l] \rightarrow \mathbb{N}$ are two functions such that $f(i) \leq g(i)$ for all $i \in[k, l]$. If $X$ is $n$-dense $(g)$ then $X$ is $n$-dense $(f)$.

Proof. One verifies the claim easily by induction on $n$.

\subsection{Upper bound}

Lemma 2 Let $f$ be non decreasing. Let $n \in \mathbb{N}$ and $X \subseteq \mathbb{N}$ be a finite set. If $X$ is $n$-dense $(f)$, then $\max X \geq F_{f, n}(\min X)$.

Proof. Being of no importance for the proof itself, we leave out the subscript $f$. Henceforth, let $x_{0}=\min X$ and $c=f\left(x_{0}\right)$. The proof goes by induction on $n$.

If $X$ is 0 -dense $(f)$, then $|X| \geq \max \left\{f\left(x_{0}\right), 3\right\}$. Thus, $\max X \geq x_{0}+2 \geq$ $F_{0}\left(x_{0}\right)$.

Secondly, assume the statement is proven for $n$ and $X$ is $(n+1)$-dense $(f)$. Consider the following partition of $X=\cup_{0 \leq i<c} Y_{i}$, where $Y_{i}$ is defined by

$$
Y_{i}=\left\{x \in X \mid F_{n}^{i}\left(x_{0}\right) \leq x<F_{n}^{i+1}\left(x_{0}\right)\right\}
$$


for $0 \leq i<c-1$ and $Y_{c-1}=\left\{x \in X \mid F_{n}^{c-1}\left(x_{0}\right) \leq x\right\}$. Now, define $G: X \rightarrow c$, as follows

$$
G(x):=i,
$$

for $x \in Y_{i}$. Since $X$ is $(n+1)$-dense $(f)$, there exists a subset $Y$ of $X$, such that $Y$ is $n$-dense $(f)$ and homogeneous for $G$. By contradiction assume $Y \subseteq Y_{i_{0}}$ for some $i_{0}$ with $0 \leq i_{0}<c-1$. The $n$-density of $Y$ and the monotonicity of $F_{n}$ yield

$$
\begin{aligned}
F_{n}^{i_{0}+1}\left(x_{0}\right)-1 & \geq \max Y_{i_{0}} \geq \max Y \geq F_{n}(\min Y) \geq \\
F_{n}\left(\min Y_{i_{0}}\right) & \geq F_{n}\left(F_{n}^{i_{0}}\left(x_{0}\right)\right)=F_{n}^{i_{0}+1}\left(x_{0}\right),
\end{aligned}
$$

a contradiction. So $Y \subseteq Y_{c-1}$, which implies

$$
\begin{aligned}
\max X & =\max Y_{c-1} \geq \max Y \geq F_{n}(\min Y) \geq \\
F_{n}\left(\min Y_{c-1}\right) & \geq F_{n}\left(F_{n}^{c-1}\left(x_{0}\right)\right)=F_{n}^{c}\left(x_{0}\right)=F_{n}^{f\left(x_{0}\right)}\left(x_{0}\right)=F_{n+1}\left(x_{0}\right),
\end{aligned}
$$

by the $n$-density of $Y$. This concludes the induction argument.

Definition 2 Define $\operatorname{PHP}_{f}: \mathbb{N} \rightarrow \mathbb{N}$ by

$$
\operatorname{PHP}_{f}(n):=\min \left\{n^{\prime} \in \mathbb{N} \mid\left[n, n^{\prime}\right] \text { is } n \text {-dense }(f)\right\} .
$$

Let $f(i)=i^{\frac{1}{A_{\omega}^{-1}(i)}}$, where $A_{\omega}$ denotes the Ackermann fuction. Then $F_{f}$ is Ackermannian, due to Theorem 1 in [OW09]. If $f$ would be non decreasing then Lemma 2 would yield

$$
\operatorname{PHP}_{f}(n) \geq F_{f, n}(n)=F_{f}(n),
$$

for all $n \in \mathbb{N}$, hence also $\mathrm{PHP}_{f}$ would Ackermannian. Since the provably total functions of I $\Sigma_{1}$ are exactly the primitive recursive functions, we would immediately obtain that $\operatorname{PHP}_{f}$ would not be provably recursive in $\mathrm{I} \Sigma_{1}$. We now show how to overcome this problem.

Theorem 1 If $f(i)=i^{\frac{1}{A_{\omega}^{-1}(i)}}$, then

$$
\mathrm{I} \Sigma_{1} \nvdash(\forall n)(\forall a)(\exists b)([a, b] \text { is } n \text {-dense }(f)) .
$$

Proof. Let $p(n):=4+3^{n+1}+(n+1)^{n+1}$ and let $f_{k}(i):=i^{\frac{1}{k}}$. It suffices to show that $\operatorname{PHP}_{f}(p(n))>A_{\omega}(n)$ Assume that $\operatorname{PHP}_{f}(p(n)) \leq A_{\omega}(n)$. Then for $i \leq$ $A_{\omega}(n)$ one has that $A_{\omega}^{-1}(i) \leq n$ which yields $f(i) \geq f_{n}(i)$ for all $i \leq \operatorname{PHP}_{f}(p(n))$. The proof of Claim 2.12 from [KLOW08] yields $F_{f_{n+1}, n+n^{2}+4 n+5}\left(p(n)>A_{\omega}(n)\right.$. Together with Lemma 1 this yields

$$
\operatorname{PHP}_{f}(p(n)) \geq \operatorname{PHP}_{f_{n}}(p(n)) \geq F_{f_{n+1}, n+n^{2}+4 n+5}(p(n))>A_{\omega}(n)
$$

which is a contradiction. 


\subsection{Lower bound}

Let $f(i)=i^{\frac{1}{A_{d}^{-1}(i)}}$, where $A_{d}$ denotes the $d$-th branch of the Ackermann function $A_{\omega}$. This function is not weakly increasing on its domain. For $i \in\left[A_{d}(k), A_{d}(k+\right.$ 1) - 1] one has that $A_{d}^{-1}(i)=k$ and on such intervals $f$ will be non decreasing. In intervals of the form $i \in\left[A_{d}(k)-1, A_{d}(k)\right]$ the function $A_{d}^{-1}$ jumps from $k-1$ to $k$. But since the intervals of the form $\left[A_{d}(k), A_{d}(k+1)-1\right]$ are rather long it is very easy to find enough points $b$ such that $f(b) \geq f(i)$ for all $i \leq b$. One simply has to choose $b$ so large that $f$ majorizes $f(c)$ where $c$ is the initial point of a last jump interval which comes before $b$. With this caveat we can consider $f$ basically as non decreasing function although it in fact is not.

Theorem 2 If $f(i)=i^{\frac{1}{A_{d}^{-1}(i)}}$, then

$$
\mathrm{I} \Sigma_{1} \vdash(\forall n)(\forall a)(\exists b)([a, b] \text { is } n \text {-dense }(f)) .
$$

Proof. Assume that $n$ and $a$ are given. Put $b:=2^{A_{d}\left(a 2^{n+1}\right) 2^{n+1}}$. Then $f(i) \leq f(b)$ for all $i \leq b$. We claim that any $Y \subseteq[a, b]$ with $|Y|>2^{A_{d}\left(a 2^{n+1}\right) 2^{k}}$ is $k$-dense $(f)$. To prove the claim we proceed by induction on $k$.

Assume the claim holds for $k-1$ and consider $Y \subseteq[a, b]$ with $|Y|>$ $2^{A_{d}\left(a 2^{n+1}\right) 2^{k}}$. Since $2^{A_{d}\left(a 2^{n+1}\right) 2^{n+1}}>A_{d}\left(2^{n+1}\right)$, we have

$$
\begin{array}{r}
f(\min Y)<f(b)=\left(2^{A_{d}\left(a 2^{n+1}\right) 2^{n+1}}\right)^{\frac{1}{A_{d}^{-1}\left(2^{A_{d}\left(a 2^{n+1}\right) 2^{n+1}}\right)}} \\
\leq\left(2^{A_{d}\left(a 2^{n+1}\right) 2^{n+1}}\right)^{\frac{1}{A_{d}^{-1}\left(A_{d}\left(2^{n+1}\right)\right)}} \\
=\left(2^{A_{d}\left(a 2^{n+1}\right) 2^{n+1}}\right)^{\frac{1}{2^{n+1}}}=2^{A_{d}\left(a 2^{n+1}\right)} \leq 2^{A_{d}\left(a 2^{n+1}\right) 2^{k-1}} .
\end{array}
$$

Let $c=f(\min Y)$ and $G: Y \rightarrow c$ be any function. Consider the partition of $Y$ induced by $G$, i.e.

$$
Y=\cup_{0 \leq i<c} Y_{i},
$$

with $Y_{i}=\{y \in Y \mid G(y)=i\}$. By contradiction, assume that $\left|Y_{i}\right| \leq 2^{A_{d}\left(a 2^{n+1}\right) 2^{k-1}}$ for every $0 \leq i<c$. Then

$$
\begin{array}{r}
2^{A_{d}\left(a 2^{n+1}\right) 2^{k}}<|Y| \leq c \cdot 2^{A_{d}\left(a 2^{n+1}\right) 2^{k-1}}=f(\min Y) \cdot 2^{A_{d}\left(a 2^{n+1}\right) 2^{k-1}} \\
<2^{A_{d}\left(a 2^{n+1}\right) 2^{k-1}} \cdot 2^{A_{d}\left(a 2^{n+1}\right) 2^{k-1}}=2^{A_{d}\left(a 2^{n+1}\right) 2^{k-1}+A_{d}\left(a 2^{n+1}\right) 2^{k-1}}=2^{A_{d}\left(a 2^{n+1}\right) 2^{k}},
\end{array}
$$

a contradiction. Thus, there exists an index $i_{0} \in\{0, \ldots, c-1\}$, such that $\left|Y_{i_{0}}\right|>$ $2^{A_{d}\left(a 2^{n+1}\right) 2^{k-1}}$. The induction hypothesis yields that $Y_{i_{0}}$ is $(k-1)$-dense $(f)$ and by definition $Y_{i_{0}}$ is homogeneous for $G$, so $Y$ is $k$-dense $(f)$.

If $k=0$ then $|Y|>2^{A_{d}\left(a 2^{n+1}\right)}>f(\min Y)$, which completes the induction argument and proves the claim.

Now return to $[a, b] .[a, b]$ is $n$-dense $(f)$ since $|[a, b]| \geq 2^{A_{d}\left(a 2^{n+1}\right) 2^{n+1}}-a \geq$ $2^{A_{d}\left(a 2^{n+1}\right) 2^{n}}$. Remarking that the function $E: \mathbb{N} \times \mathbb{N} \rightarrow \mathbb{N}$, defined by $E(a, n)=$ $2^{A_{d}\left(a 2^{n+1}\right) 2^{n+1}}$ is primitive recursive, completes the proof.

Let $\mathrm{PHP}_{f}$ stand for " $(\forall n)(\forall a)(\exists b)([a, b]$ is $n$-dense $(f))$ ". Then we obtain the following picture. 


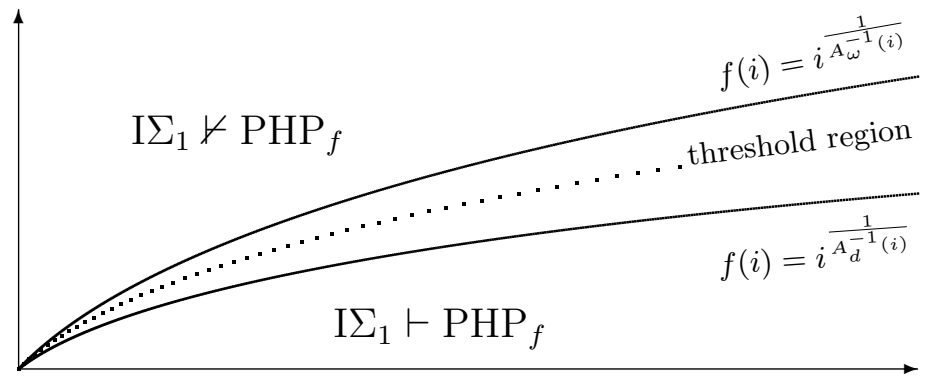

Fig. 1. Phase transition for $\mathrm{PHP}_{f}$.

\section{$3(\alpha, 2)$-Density}

In this section we work with a fixed number of colours, namely two. For a limit ordinal not exceeding $\omega^{2}$ we define the fundamental sequence as follows. We put $\omega \cdot(k+1)[n]=\omega \cdot k+n$ and we set $\omega^{2}[n]=\omega \cdot n$.

Definition $3 X$ is called $(0,2)$-dense $(f)$ if $|X| \geq \max \{f(\min X), 3\} . X$ is called $(\alpha+1,2)$-dense $(f)$ if for all $G: X \rightarrow 2$ there exists $Y \subseteq X$, such that $Y$ is $(\alpha, 2)$-dense $(f)$ and $Y$ is homogeneous for $G$. If $\lambda$ is a limit ordinal, then $X$ is called $(\lambda, 2)$-dense $(f)$ if for all $G: X \rightarrow 2$ there exists $Y \subseteq X$, such that $Y$ is $(\lambda[\min X], 2)$-dense $(f)$ and $Y$ is homogeneous for $G$.

Lemma 3 Let $k \leq l$ and $f, g:[k, l] \rightarrow \mathbb{N}$ be non decreasing such that $f(i) \leq g(i)$ for all $i \in[k, l]$. If $X \subseteq[k, l]$ is $(n, 2)$-dense $(g)$ then $X$ is is $(n, 2)$-dense $(g)$.

\subsection{Upper bound}

In this section we will use another hierarchy which we call $B_{f, \alpha}$ and which turns out to be related to $F_{f, k}$. We define $B_{\alpha}$, depending on $f$, by

$$
\begin{aligned}
B_{f, 0}(n) & :=n+1 \\
B_{f, \alpha+1}(n) & :=B_{f, \alpha}\left(B_{f, \alpha}(n)\right):=B_{f, \alpha}^{2}(n) \\
B_{f, \lambda}(n) & :=B_{f, \lambda[f(n)]}(n),
\end{aligned}
$$

for all $n \in \mathbb{N}$ and ordinals $\alpha$ and $\lambda$, with the latter a limit ordinal. As for $F_{f}$, we leave out the subscript $f$ and write $B_{\alpha}$ if it is clear which $f$ we are working with. We first show a simple lemma concerning the relation between the two hierarchies defined in this paper. This lemma might be considered as folklore.

Lemma 4 Let $k, l$ and $m$ be natural numbers. Then $B_{f, \omega \cdot k+l}(m)=F_{2^{f}, k}^{2^{l}}(m)$.

Proof. We proceed by main induction on $k$ and subsidiary induction on $l$. If $k$ equals $l$ equals zero, we have $B_{f, 0}(m)=m+1=F_{2^{f}, 0}(m)$. 
Assume the statement is proven for $k-1$, we will prove it for $k$ by subsidiary induction on $l$.

If $l=0$, then the main induction hypothesis yields

$$
B_{f, \omega \cdot(k-1)}(m)=F_{2^{f}, k-1}(m) .
$$

Assume the claim is proven for $l-1$. We have

$$
\begin{aligned}
B_{f, \omega \cdot(k-1)+l}(m) & =B_{f, \omega \cdot(k-1)+l-1}\left(B_{f, \omega \cdot(k-1)+l-1}(m)\right) \\
= & F_{2^{f}, k-1}^{2^{-1}}\left(F_{2^{f}, k-1}^{2^{l-1}}(m)\right)=F_{2^{f}, k-1}^{2^{l}}(m),
\end{aligned}
$$

which proves the statement for $k-1$ and every $l$. Using this fact, we obtain

$$
\begin{array}{r}
\left.\left.B_{f, \omega \cdot k}(m)=B_{f, \omega \cdot(k-1)+\omega[f(m)]}(m)\right)=B_{f, \omega \cdot(k-1)+f(m)}(m)\right) \\
=F_{2^{f}, k-1}^{2^{f(m)}}(m)=F_{2^{f}, k}(m),
\end{array}
$$

which concludes the main induction and proves the statement.

Lemma 5 Let $f$ be non decreasing. Let $n$ be a natural number and $\alpha$ be any ordinal. If $X \subseteq \mathbb{N}$ is $(\alpha, 2)$-dense $(f)$, then $\max X \geq B_{f, \alpha}(\min X)$.

Proof. Being of no importance for the proof itself, we leave out the subscript $f$. Henceforth, let $x_{0}=\min X$. The proof goes by transfinite induction on $\alpha$.

If $X$ is $(0,2)$-dense $(f)$, then $|X| \geq \max \left\{f\left(x_{0}\right), 3\right\}$. Thus, $\max X \geq x_{0}+2>$ $x_{0}+1=B_{0}\left(x_{0}\right)$.

Assume the statement is proven for $\alpha$ and $X$ is $(\alpha+1,2)$-dense $(f)$. Define $G: X \rightarrow 2$ as follows

$$
G(x):=\left\{\begin{array}{ll}
0 & \text { if } x_{0} \leq x<B_{\alpha}\left(x_{0}\right) \\
1 & \text { if } B_{\alpha}\left(x_{0}\right) \leq x
\end{array},\right.
$$

for all $x \in X$. Since $X$ is $(\alpha+1,2)$-dense $(f)$, there exists a subset $Y$ of $X$, such that $Y$ is $(\alpha, 2)$-dense $(f)$ and $Y$ is homogeneous with respect to $G$. By contradiction, assume $G$ takes colour 0 on $Y$. Then, by the induction hypothesis,

$$
B_{\alpha}\left(x_{0}\right)-1 \geq \max Y \geq B_{\alpha}(\min Y)=B_{\alpha}\left(x_{0}\right),
$$

a contradiction. So, the colour needs to be 1 , which implies

$$
Y \subseteq\left\{x \in X \mid B_{\alpha}\left(x_{0}\right) \leq x\right\} .
$$

The induction hypothesis yields

$$
\max X \geq \max Y \geq B_{\alpha}(\min Y)=B_{\alpha}\left(B_{\alpha}\left(x_{0}\right)\right)=B_{\alpha+1}\left(x_{0}\right) .
$$

Finally, assume the statement is proven for all $\alpha<\lambda$, with $\lambda$ a limit ordinal, and $X$ is $(\lambda, 2)$-dense $(f)$. There exists a subset $Y$ which is $\left(\lambda\left[f\left(x_{0}\right)\right], 2\right)$-dense $(f)$. We obtain by the induction hypothesis

$$
\max X \geq \max Y \geq B_{\lambda\left[f\left(x_{0}\right)\right]}(\min Y) \geq B_{\lambda\left[f\left(x_{0}\right)\right]}\left(x_{0}\right)=B_{\lambda}\left(x_{0}\right) .
$$

This completes the proof. 
Definition 4 Define $\mathrm{PHP}_{f}: \mathbb{N} \rightarrow \mathbb{N}$ by

$$
\operatorname{PHP} 2_{f}(n):=\min \left\{n^{\prime} \in \mathbb{N} \mid\left[n, n^{\prime}\right] \text { is }\left(\omega^{2}, 2\right) \text {-dense }(f)\right\} .
$$

Fix $f(i)=\frac{1}{A_{\omega}^{-1}(i)} \log (i)$ for the rest of this subsection.

Lemma 6 Let $f_{n}(i):=\frac{1}{n} \cdot \log _{2}(i)$. Then $\mathrm{PHP} 2_{f_{n}}\left(2^{n^{2}}\right) \geq F_{2^{f_{n}}}(n)$ holds for every $n \in \mathbb{N}$.

Proof. Let $X=\left[2^{n^{2}}, \operatorname{PHP} 2_{f}\left(2^{n^{2}}\right)\right]$. Define $G: X \rightarrow 2$ by $G(x)=0$ for every $x \in X$. Since $X$ is $\left(\omega^{2}, 2\right)$-dense $\left(f_{n}\right)$ there exists $Y \subseteq X$, such that $Y$ is $\left(\omega^{2}\left[f_{n}(\min X)\right], 2\right)$-PHP2-dense $\left(f_{n}\right)$, i.e. $\left(\omega \cdot f_{n}\left(2^{n^{2}}\right), 2\right)$-dense $\left(f_{n}\right)$. Lemma 4 and Lemma 5 yield

$$
\begin{aligned}
\operatorname{PHP} 2\left(2^{n^{2}}\right) & \geq \max Y \geq B_{f_{n}, \omega \cdot f(\min Y)}(\min Y) \geq B_{f_{n}, \omega \cdot f\left(2^{n^{2}}\right)}\left(2^{n^{2}}\right) \\
& =F_{2^{f_{n}, f_{n}\left(2^{n^{2}}\right)}}\left(2^{n^{2}}\right) \geq F_{2^{f_{n}, n}}(n)=F_{2^{f_{n}}}(n),
\end{aligned}
$$

since $f_{n}\left(2^{n^{2}}\right)=n$.

Corollary 1 If $f(i)=\frac{1}{A_{\omega}^{-1}(i)} \log (i)$, then

$$
\mathrm{I} \Sigma_{1} \nvdash(\forall a)(\exists b)\left([a, b] \text { is }\left(\omega^{2}, 2\right) \text {-dense }(f)\right) \text {. }
$$

Proof. Let $p(n)=4+3^{n+1}+(n+1)^{n+1}$ and $f_{k}(i):=\frac{1}{k} \log _{2}(i)$. It suffices to show that PHP2 $2_{f}\left(2^{p(n)^{2}}\right)>A_{\omega}(n)$. Assume for a contradiction that PHP2 $2_{f}\left(2^{p(n)^{2}}\right) \leq$ $A_{\omega}(n)$. For $i \leq A_{\omega}(n)$ one has $A_{\omega}^{-1}(i) \leq n$ hence $f(i) \geq f_{n}(i)$ for all $i \leq$ PHP2 $f\left(2^{p(n)^{2}}\right)$. This yields PHP2 $2_{f}\left(2^{p(n)^{2}}\right) \geq \operatorname{PHP} 2_{f_{n}}\left(2^{p(n)^{2}}\right) \geq F_{2^{f_{n}}}(p(n)>$ $A_{\omega}(n)$. Contradiction!

\subsection{Lower bound}

As in Section 2.2 let $f(i)=\frac{1}{A_{d}^{-1}(i)} \log (i)$, where $A_{d}$ denotes the $d$ th branch of the Ackermann function $A_{\omega}$. Recall from Section 2.2 that $f$ is almost non decreasing and that it is easy to identify the jumps for $f$.

Theorem 3 If $f(i)=\frac{1}{A_{d}^{-1}(i)} \log (i)$, then

$$
\mathrm{I} \Sigma_{1} \vdash(\forall a)(\exists b)\left([a, b] \text { is }\left(\omega^{2}, 2\right) \text {-dense }(f)\right) .
$$

Proof. Assume that $a$ is given. Put $b:=2^{A_{d}\left(2^{a+2}\right) 2^{a+1}}$. Then $f(i) \leq f(b)$ for all $i \leq b$. We claim that any $Y \subseteq[a, b]$, with $|Y|>2^{A_{d}\left(2^{a+2}\right) 2^{k}}$ is $(\omega \cdot k, 2)$-dense $(f)$. The proof goes by induction on $k$.

Let $k=0$. Since $2^{A_{d}\left(2^{a+2}\right) 2^{a+1}}>A_{d}\left(2^{a+2}\right)$, we have

$$
\begin{array}{r}
f(\min Y)<f(b)=\frac{1}{A_{d}^{-1}\left(2^{A_{d}\left(2^{a+2}\right) 2^{a+1}}\right)} \log \left(2^{A_{d}\left(2^{a+2}\right) 2^{a+1}}\right) \\
\quad<\frac{1}{2^{a+2}} A_{d}\left(2^{a+2}\right) 2^{a+1}<A_{d}\left(2^{a+2}\right),
\end{array}
$$


so, $|Y|>2^{A_{d}\left(2^{a+2}\right)}>\max \{f(\min Y), 3\}$, i.e. $Y$ is $(0,2)$-dense $(f)$.

Assume the assertion holds for $k-1$ and consider $Y \subseteq[a, b]$ with $|Y|>$ $2^{A_{d}\left(2^{a+2}\right) 2^{k}}$. We claim that if $Z \subseteq Y$ and $|Z|>2^{A_{d}\left(2^{a+2}\right) 2^{k-1}+l}$, then $Z$ is $(\omega$. $(k-1)+l, 2)$-dense $(f)$. The proof goes by subsidiary induction on $l$.

If $l=0$, then the claim follows by the main induction hypothesis. Assume the claim holds for $l-1$ and $|Z|>2^{A_{d}\left(2^{a+2}\right) 2^{k-1}+l}$. Let $G: Z \rightarrow 2$ be any function. Consider the partition of $Z$ induced by $G$, i.e.

$$
Z=Z_{0} \cup Z_{1},
$$

with $Z_{i}=\{z \in Z \mid G(z)=i\}$. By contradiction, assume that

$$
\left|Z_{i}\right| \leq 2^{A_{d}\left(2^{a+2}\right) 2^{k-1}+l-1},
$$

for $i=0,1$. Then

$$
2^{A_{d}\left(2^{a+2}\right) 2^{k-1}+l}<|Z| \leq 2 \cdot 2^{A_{d}\left(2^{a+2}\right) 2^{k-1}+l-1}=2^{A_{d}\left(2^{a+2}\right) 2^{k-1}+l},
$$

a contradiction. Thus, there exists an index $i_{0} \in\{0,1\}$, such that $\left|Z_{i_{0}}\right|>$ $2^{A_{d}\left(2^{a+2}\right) 2^{k-1}+l-1}$. The induction hypothesis yields $Z_{i_{0}}$ is $(\omega \cdot(k-1)+l-1,2)$ dense $(f)$, and so $Z$ is $(\omega \cdot(k-1)+l, 2)$-dense $(f)$, since $Z_{i_{0}}$ is homogeneous for $G$. This proves the latter claim.

Now return to $Y$. Let $G: Y \rightarrow 2$ be any function. Consider the partition of $Y$ induced by $G$, i.e.

$$
Y=Y_{0} \cup Y_{1},
$$

with $Y_{i}=\{y \in Y \mid G(y)=i\}$. In the same way as above, one can prove there exists an index $i_{0} \in\{0,1\}$, such that

$$
\left|Y_{i_{0}}\right|>2^{A_{d}\left(2^{a+2}\right) 2^{k}-1}=2^{A_{d}\left(2^{a+2}\right) 2^{k-1}+A_{d}\left(2^{a+2}\right) 2^{k-1}-1} .
$$

Since

$$
A_{d}\left(2^{a+2}\right) 2^{k-1} \geq A_{d}\left(2^{a+2}\right) \geq f(\min Y)+1,
$$

we have $\left|Y_{i_{0}}\right|>2^{A_{d}\left(2^{a+2}\right) 2^{k-1}+f(\min Y)}$. The latter claim yields $Y_{i_{0}}$ is $(\omega \cdot(k-$ $1)+f(\min Y), 2)$-dense $(f)$, i.e. $(\omega \cdot k[f(\min Y)], 2)$-dense $(f)$. Thus $Y$ is $(\omega \cdot k, 2)$ dense $(f)$, since $Y_{i_{0}}$ is homogeneous for $G$.

We finally prove that $[a, b]$ is $\left(\omega^{2}, 2\right)$-dense $(f)$. Let $G:[a, b] \rightarrow 2$ be any function and consider the partition of $[a, b]$ induced by $G$, i.e.

$$
[a, b]=Y_{0} \cup Y_{1},
$$

with $Y_{i}=\{y \in[a, b] \mid G(y)=i\}$. Remark that $|[a, b]|>2^{A_{d}\left(2^{a+2}\right) 2^{a+1}}-a \geq$ $2^{A_{d}\left(2^{a+2}\right) 2^{a}+1}$. Similarly as before, there exists an index $i_{0} \in\{0,1\}$, such that

$$
\left|Y_{i_{0}}\right|>2^{A_{d}\left(2^{a+2}\right) 2^{a}} \geq 2^{A_{d}\left(2^{a+2}\right) 2^{f(a)}} .
$$

The main claim yields $Y$ is $(\omega \cdot f(a), 2)$-dense $(f)$, i.e. $\left(\omega^{2}[f(a)], 2\right)$-dense $(f)$. In combination with $Y_{i_{0}}$ being homogeneous for $G$, this implies $[a, b]$ is $\left(\omega^{2}, 2\right)$ dense $(f)$. 


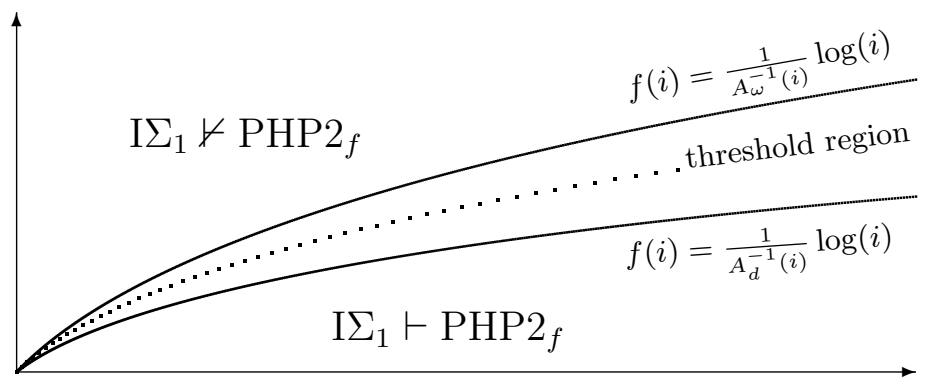

Fig. 2. Phase transition for $\mathrm{PHP} 2_{f}$.

Let PHP2 $2_{f}$ stand for " $(\forall n)(\forall a)(\exists b)\left([a, b]\right.$ is $\left(\omega^{2}, 2\right)$-dense $\left.(f)\right)$ ". Once again, we obtain the following picture.

In accordance with the referees (for which we are grateful for valuable comments) we expect that it will be not too hard to show that for natural choices of $f$ the principles $\mathrm{PHP}_{\log \text { of }}$ and $\mathrm{PHP} 2_{f}$ are equivalent over $\mathrm{I} \Sigma_{1}$.

\section{References}

[DSW08] Michiel De Smet and Andreas Weiermann: Phase Transitions for Weakly Increasing Sequences. Proceedings of CiE 2008, (2008) 168-174.

[DS11] Michiel De Smet : Unprovability and phase transitions in Ramsey theory. PhD thesis, Ghent 2011.

[Hir87] Jeffry Lynn Hirst. Combinatorics in subsystems of second-order arithmetic. PhD thesis, Pennsylvania State University, 1987.

[KLOW08] Menachem Kojman, Gyesik Lee, Eran Omri and Andreas Weiermann: Sharp thresholds for the phase transition between primitive recursive and Ackermannian Ramsey numbers JCT A. 115(6). (2008), p.1036-1055

[OW09] Eran Omri and Andreas Weiermann. Classifying the phase transition threshold for Ackermannian functions. Ann. Pure Appl. Logic, 158(3):156$162,2009$.

[WVH2012] Andreas Weiermann and Wim Van Hoof: Sharp phase transition thresholds for the Paris Harrington Ramsey numbers for a fixed dimension. Proc. Amer. Math. Soc. 140 (2012), no. 8, 29132927.

[Par78] Jeff B. Paris. Some independence results for Peano arithmetic. J. Symbolic Logic, 43(4):725-731, 1978. 